\title{
Atención, memoria y funciones ejecutivas en los trastornos del espectro autista: ¿cuánto hemos avanzado desde Leo Kanner?
}

Attention, memory and executive functions in Autism Spectrum Disorders. How much do we go forward since Leo Kanner?

\section{Raquel Seijas Gómez ${ }^{\text {a }}$.}

${ }^{a}$ Psicóloga Clínica. Neuropsicóloga. Palma de Mallorca, España.

Correspondencia: Raquel Seijas Gómez(raquelseijasgomez@gmail.com)

Recibido: 13/11/2014; aceptado: 22/03/2015

RESUMEN: Los trastornos del espectro autista se caracterizan por dificultades en la comunicación social y patrones de conducta e intereses restringidos y repetitivos. Desde la descripción pionera de Leo Kanner a mediados del S. XX y que aludía de forma indirecta a posible afectación en algunas funciones cognitivas como lenguaje o funciones ejecutivas, se han publicado numerosos trabajos relativos a la fenomenología, la etiología y el tratamiento; y desde disciplinas variadas como la Psicología Cognitiva y la Neuropsicología, entre otras. La revisión de los trabajos publicados en los últimos 14 años relativos a la evaluación de la memoria, la atención y las funciones ejecutivas en niños y adolescentes con trastornos del espectro autista permite concluir un perfil de afectación particular, que sostiene y precisa en mayor medida las hipótesis que Kanner había propuesto en el siglo pasado.

PALABRAS CLAVE: Trastorno autístico. Atención. Memoria. Función ejecutiva.
ABSTRACT:Autism spectrum disorders are characterized by difficulties in social communication as wella as restricted and repetitive behavior patterns and interests. Since Leo Kanner's first description in the mid-20th century, which indirectly mentioned impairments in some cognitive functions as language or executive funciones, many papers concerning phenomenology, etiology and treatment have been publicated; from different disciplines as Cognitive Psychology and Neuropsychology, among others. The review of the articles published in the last 14 years related to the evaluation of memory, attention and executive functions in autism spectrum disorders allow us to conclude an impaired cognitive profile that supports and specifies Kanners's hypothesis in the last century.

KEY WORDS: Autistic Disorder. Attention. Memory. Executive Function.

De Leo Kanner al DSM-5: pasado y presente de los Trastornos del Espectro Autista.

No fue hasta el año 1943 cuando el psiquiatra austríaco Leo Kanner describió el autismo como un síndrome, y lo diferenció del concepto de síntoma de alejamiento de la realidad externa propio de los cuadros de esquizofrenia que había propuesto Bleuler. Al mismo tiempo, Kanner lo conceptualiza como cuadro único y claramente diferenciable de la esquizofrenia u otros trastornos. En su artículo titulado "Autistics disturbances of affective contact" recoge una serie de observaciones clínicas de 11 niños y adolescentes, realizadas entre 1938 y 1943, que presentaban unas características peculiares, que se enumeran a continuación (1): 1) retraso en la adquisición 
del lenguaje y uso infrecuente del mismo (ecolalia), 2) "excelente memoria de repetición" (tanto para palabras como para frases), 3) "deseo obsesivo de invariabilidad" (malestar ante cualquier cambio en el ambiente o ante ruidos fuertes $u$ objetos en movimiento; característica que califica como nuclear tras ampliar el número de observaciones clínicas (2), 4) "limitaciones en la variedad de actividades espontáneas" (necesidad de repetir la misma rutina de acciones o verbalizaciones), 5) dificultad para iniciar interacciones sociales mostrando preferencia por las actividades solitarias, y 6) ausencia de contacto ocular en las situaciones de interacción social. Kanner les atribuye además una elevada inteligencia (basándose en la capacidad de los menores para reproducir oraciones complejas o secuencias de números de forma literal y sin llegar tener una medida más objetiva resultado de alguna prueba psicométrica). La carencia de afectividad en las interacciones de los progenitores con los menores llamó también la atención de este psiquiatra austríaco, y le llevó a plantearse si podía haber alguna relación causal entre esta falta de afectividad y las peculiaridades de sus pacientes. Un planteamiento similar, aunque más extremo, fue defendido por Bruno Bettelheim en su teoría de las "madres nevera" (3) al igual que por otro autores de enfoque psicoanalítico; sin embargo Kanner fue más allá y asumió finalmente la idea de autismo como lo que hoy en día conceptualizamos como un trastorno del neurodesarrollo (4), al entender las manifestaciones conductuales observadas en los pacientes como "componentes constitucionales de la respuesta emocional" (1).

Un año después de Kanner, y desde el continente Europeo, Hans Asperger hacía también sus aportaciones en la fenomenología del autismo con la descripción de los llamados "pequeños profesores": pacientes carentes de empatía, ingenuidad, poca habilidad para hacer amigos, lenguaje pedante o repetitivo, pobre comunicación no verbal, interés desmesurado por ciertos temas y capacidad para dar todo tipo de detalles respecto a éstos, y torpeza motora (4); sintomatología que más tarde se englobaría bajo el diagnóstico de Trastorno de Asperger. Otra aportación muy influyente en la idea actual de estos trastornos es la de Lorna Wing y Judith Gould. A partir de los resultados de su estudio observacional en un área de Londres estas dos autoras proponen en 1979 una concepción diferente del autismo entendiéndolo como un continuum de sintomatología dentro del "espectro autista", al percatarse de la dificultad de etiquetar como autistas a algunos casos que únicamente compartían algunas de las características, o con un diferente nivel de gravedad de los síntomas (5).

El vigente DSM-5 parte del concepto de espectro autista, elimina los subtipos de Trastornos Generalizados del desarrollo establecidos en la anterior versión y mantiene como síntomas nucleares la alteración en la comunicación social y las actividades relacionadas con ésta, además de patrones de conductas, intereses o actividades restrictivas y repetitivas. Incluye especificadores del nivel de gravedad y de comorbilidad con déficits en lenguaje, deficiencia intelectual, otra condición médica, genética o ambiental conocida, o catatonía (6). Estos síntomas recogidos en el ma- 
ORIGINALES Y REVISIONES

nual son algunos de los descritos por Kanner en 1943, y el planteamiento de autismo como trastorno del neurodesarrollo podría haber sido avanzado también por este autor al entender las manifestaciones conductuales observadas en los pacientes como "componentes constitucionales de la respuesta emocional" (4), tal y como se ha mencionado previamente. Sin embargo, se quiere destacar que el listado de síntomas descrito por Kanner y mantenido en el DSM V hace referencia a funciones como lenguaje, flexibilidad conductual o dificultades en la interacción social; sin incluir otras funciones como memoria (a la que Kanner calificaba como excelente en contextos de repetición de material complejo), la atención y otras funciones ejecutivas, siendo todas ellas fundamentales para comprender de un modo más amplio la fenomenología de los trastornos del espectro autista y las observaciones que despertaron el interés del médico austríaco.

Neuropsicología y psicología cognitiva en losTrastornos del Espectro Autista:

Desde el campo de la Neuropsicología y en un intento de comprender la sintomatología propia de los trastornos del espectro autista como resultado de una alteración cerebral, Damasio y Maurer establecen una similitud entre esta sintomatología y la observada en casos de daño cerebral adquirido (tanto en humanos como en animales), mediada por una disfunción dopaminérgica en las conexiones entre córtex mesolímbico, los ganglios basales y el tálamo (7). Estudios más recientes con técnicas de neroimagen estructural y funcional corroboran la presencia de patrones de activación alterados en el giro fusiforme, córtex cingulado anterior y córtex temporal, conexiones subcorticales (amígdala-hipocampo), giro frontal inferior (área de Broca), córtex prefrontal y temporo-parietal, área de Wernicke y lóbulo parietal posterior, cingulado posterior y cerebelo; todos ellos durante la realización de determinadas tareas lingüísticas, sociales y durante la realización de las conductas estereotipadas y repetitivas (8). Por otra parte, no parece haber un consenso respecto a la presencia o no de alteraciones estructurales en áreas como el cerebelo o la corteza límbica, o la reducción de sustancia gris o de sustancia blanca (8-9). Hay que destacar, sin embargo, que la práctica totalidad de los estudios de neuroimagen en los trastornos del espectro autista emplean muestras adultas, y sus conclusiones no se pueden aplicar a población infantil dado que no tienen en cuenta la variable de desarrollo cerebral.

Otros autores plantean que la sintomatología característica de los TEA puede entenderse en el marco de déficits cognitivos o alteración en el patrón de procesamiento de la información, sin llegar a establecer correlatos de alteraciones estructurales. Desde un enfoque más cercano a la psicología cognitiva y los modelos de procesamiento de la información, Utah Frith propone la Teoría de la Coherencia 
central, partiendo de varios trabajos y de las hipótesis ya planteadas por Hemerlin y $\mathrm{O}^{\prime}$ Connor sobre la alteración en la integración de la información. Baron-Cohen defiende también con la Teoría de la Mente la dificultad que presentan estos pacientes a la hora de representar los estados mentales propios o ajenos y que explicarían las dificultades en situaciones de interacción social (10). La hipótesis de disfunción ejecutiva y de déficits en la cognición social es la que más investigación ha generado en los TEA. Sin embargo, se puede decir que el estudio de otras funciones cognitivas básicas, como la atención o la memoria, sin olvidar a las funciones ejecutivas, puede ser un aspecto clave que ayude a entender en las dificultades que presentan estos pacientes, además de complementar los planteamientos de estas teorías.

"Ausencia de contacto ocular en las interacciones sociales": la atención en los Trastornos del Espectro Autista.

La atención es una función compleja que nos permite seleccionar la información relevante y mantener ésta hasta alcanzar nuestro objetivo, además de básica para varios procesos cognitivos y para el aprendizaje. Las alteraciones atencionales no son exclusivas de ningún trastorno en particular, sino que están presentes en varios cuadros neurológicos y psiquiátricos.

Partiendo del modelo atencional de Sholberg y Mateer $(11)^{1}$, varios trabajos encuentran problemas en atención focalizada y selectiva en niños con TEA, en comparación con sujetos controles de la misma edad, nivel de inteligencia y nivel de desarrollo lingüístico (12). En un intento de precisar la adecuación de la respuesta a los requisitos de la tarea, el diseño experimental de varios estudios incluye sofisticados aparatos de medida de la trayectoria ocular; y encuentran también una mayor resistencia a la hora de cambiar el foco atencional hacia otros estímulos, y un mayor tiempo de focalización en estímulos no sociales y no relacionados con intereses restringidos (13), así como también en aquellos relacionados (14-18). La dificultad para hacer un "desenganche" atencional es, para algunos autores, un marcador fenotípico de TEA en edades tempranas. Sin descartar alteraciones debido a la ausencia de grupo control, Johnson et al (2007) encuentran un rendimiento superior en tareas de atención sostenida en comparación con menores con diagnóstico de Trastorno por Déficit de Atención e Hiperactividad (19), y, tal y como cabría esperar, aquellos casos de comorbilidad diagnóstica mostraban un perfil cognitivo más patológico (20-22).

\footnotetext{
M. M. Sholberg y C. A. Mateer defienden un modelo atencional, con enfoque clínico, en el que conciben la atención como un sistema jerárquico de cinco componentes, y en el que la preservación de los subtipos inferiores es requisito imprescindible para el correcto funcionamiento de los superiores. En orden de menor a mayor estos componentes son: atención focalizada, sostenida, selectiva, alternante y dividida.
} 
ORIGINALES Y REVISIONES

Otros estudios relativos a atención focalizada y selectiva destacan que los niños con TEA tienen dificultades en el procesamiento de estímulos sociales como rostros u otras partes del cuerpo claves en situaciones de interacción personal y estas dificultades quedan son más evidentes en las interacciones reales que en situaciones de laboratorio (23-24). Jarrold et al (2013) emplean un entorno de realidad virtual en el que los menores con diagnóstico de TEA no mostraban un rendimiento inferior al de los controles en la focalización en estímulos sociales, pero las dificultades aparecían cuando se introducía la consigna de mantener una conversación (25). La preferencia manifiesta por estímulos no sociales no parece responder a una deficiente exploración de los rostros (es decir, al igual que los niños sin este diagnóstico, mantienen foco atencional durante más tiempo en la zona de los ojos y de la boca) (26-27); sin embargo sí muestran algunas peculiaridades a la hora de analizar las caras de otras personas, como la fijación en estímulos menos discriminativos en tareas de comparación (28) y en aquellos que no proporcionan información de carácter emocional (29). En cuanto a los últimos subtipos atencionales de la jerarquía de Sholberg y Mateer, no se han encontrado trabajos centrados en atención alternante y dividida, aunque partiendo del planteamiento de las autoras cabría esperar que si los niveles inferiores atencionales están afectados es muy probable que presenten dificultades en las tareas que implican demandas atencionales más complejas y participación de las funciones ejecutivas, como se verá más adelante.

Para finalizar este punto referente a la atención, se hace alusión a una respuesta atencional que sí bien no está incluida en ningún modelo atencional, es clave para entender las interacciones sociales: la atención conjunta, y que había llamado la atención de Kanner al percatarse de la ausencia de contacto ocular que mostraban sus pacientes durante las interacciones sociales. La atención conjunta es la habilidad de responder a la dirección de la mirada del otro con finalidad comunicativa, y también la iniciativa por parte del niño en la búsqueda de atención en el otro (a través de la mirada, el señalamiento o muestra de objetos) y que frecuentemente se verbaliza en forma de protoimperativos y protodeclarativos. Los niños comienzan a emplear la dirección de la mirada, alternándola entre el objeto deseado y el adulto, alrededor del final del primer año de vida. Aunque los niños con TEA pueden llegar a emplear algunas formas de señalamiento y demostrar cierto uso de los protoimperativos, con el objetivo de alcanzar el objeto deseado y no con finalidad social, no llegan a alcanzar un desarrollo normal al observado en pares sin este diagnóstico (30). La atención conjunta parece estar relacionada con el desarrollo posterior cognitivo y con el lingüístico (31-32); y, específicamente, con el del lenguaje comprensivo (33). Varios trabajos destacan también la importancia de la atención conjunta como marcador diferencial entre sujetos con TEA y sujetos con trastorno específico del lenguaje (23), con menores con diagnóstico de retraso madurativo (34), y también con sujetos sin diagnóstico psiquiátrico o neuropsicológico (35-36), llegando a describirse como un 
marcador temprano detectable ya alrededor del primer año de vida (37), o como un componente del fenotipo de TEA en niños con antecedentes familiares de este trastorno (36). Con el objetivo de estudiar la respuesta de atención conjunta, algunos trabajos describen situaciones artificiales (diseños virtuales que simulan condiciones de atención conjunta), mientras que otros la analizan en interacciones reales, obteniéndose resultados opuestos. Por una parte, algunos trabajos ponen de manifiesto que los sujetos con TEA cometen más errores en la identificación de los estímulos targets y parecen no emplear la facilitación de la orientación ocular del modelo cuando se emplean sujetos virtuales que indican al paciente hacia dónde dirigir su mirada (31), mientras que otros destacan la normalidad en la monitorización visual y el seguimiento de las claves de orientación visual proporcionadas $(32,37)$. En contexto de interacción real, las dificultades son más evidentes (38), teniendo en cuenta la menor complejidad de las situaciones experimentales en comparación con las situaciones de interacción real.

"Excelente memoria de repetición": la memoria en los Trastornos del Espectro Autista:

En el estudio de la memoria se han establecido tradicionalmente varias dicotomías, tomando como variables, entre otras, el formato de la información a almacenar o el tiempo de retención de la misma.

Comenzando por la fuente de entrada del material a almacenar, se han postulado dos tipos diferentes, de memoria: la memoria verbal y memoria visual. Frente a la elevada capacidad de memorización de material verbal que había llamado la atención de Leo Kanner, varios trabajos recientes ponen de manifiesto resultados opuestos en memoria verbal y visual. Por un lado, algunos autores encuentran un rendimiento similar entre niños con TEA y controles sin este diagnóstico tanto en las medidas de memoria visual como verbal a largo plazo (39-41); mientras que otros describen un rendimiento inferior en ambas modalidades, rendimiento que parece más explicado por una mala codificación de la información más que por la afectación de la fase de almacenamiento (42).

Tulving estableció una dicotomía de la memoria basándose en el contenido almacenado y las características propias de éste, y dio nombre a la memoria semántica y a la memoria episódica. En los TEA la memoria semántica parece estar relativamente preservada, al contrario que la memoria episódica (43), y sus resultados en tareas de recuerdo de eventos pasados autobiográficos son inferiores a los esperables (44-45). De forma más concreta, a la hora de recuperar eventos autobiográficos los niños y adolescentes con este diagnóstico omiten más detalles que controles sin este diagnóstico y su recuerdo es más desorganizado (46). 
Otra división clásica en psicología de la memoria distingue entre memoria declarativa o explícita y memoria no declarativa o implícita. Esta distinción se basa en la consciencia en la recuperación del material almacenado. Se considera a la memoria episódica y semántica como subtipos de memoria explícita, mientras que algunos ejemplos de memoria implícita serían el priming, las habilidades motoras y el condicionamiento motor, entre otros. Renner et al defienden la preservación de ambos tipos de memoria en los niños y adolescentes con TEA, aunque su patrón de recuperación y organización del material parece ser también particular en cuanto a que no presentan el esperable efecto de priming, así como tampoco el de primacía y el de recencia (41). Tampoco resulta significativo el mejor recuerdo de las acciones observadas frente a las realizadas (47), a pesar de los hallazgos de algunos autores a favor de este resultado (48-49).

Pasando ahora a describir lo que se ha denominado "memoria ejecutiva", los resultados de los trabajos son, una vez más, opuestos. Algunos autores encuentran un mal rendimiento en tareas de memoria de trabajo visual (42, 50-53); resultados que no parece convencer a otros autores. Así, O’Hearn et al (2008) realizan una revisión sobre las funciones ejecutivas en autismo y concluyen que los resultados opuestos de diferentes estudios se deben a los sesgos introducidos por la propia tarea experimental (o más concretamente, y en base a las hipótesis de estas autoras, a la baja fiabilidad de los test clásicos de memoria a la hora de detectar posibles déficit). Para evitar este sesgo emplean un paradigma experimental más complejo de medida de la respuesta oculomotora que, sin embargo, sigue poniendo de manifiesto un rendimiento inferior en los menores con TEA (9). En la línea de tareas más clásicas de memoria visual, otros autores concluyen que, frente al buen rendimiento en el recuerdo de targets geométricos, los niños con TEA no se benefician de las claves espaciales que facilitarían el análisis y recuerdo posterior de los estímulos, y carecen por tanto de la "capacidad de cierre" necesaria para una correcta integración de las figuras (54). En comparación con los menores con Trastorno por Déficit de Atención e Hiperactividad, en los TEA hay un mejor rendimiento en tareas de memoria de trabajo verbal (55) y también visual (56).

Otro de los subtipos de memoria que podemos considerar "ejecutiva" es la metamemoria o conocimiento sobre nuestra propia memoria y las estrategias que podemos llegar a emplear (57). Wojcik et al encuentran que la metamemoria se encuentra afectada en los TEA, pero únicamente para material episódico y no para el semántico (58), resultados que respaldarían la hipótesis de disociación en el rendimiento entre ambos subtipos de memoria explícita, tal y como se ha descrito previamente.

Frente a esta variedad de resultados, cabe señalar que uno de las conclusiones más citada en varios trabajos es que los niños con TEA no emplean de forma espontánea estrategias de categorización semántica que facilitarían el almacenamiento y la recuperación de la información (40-42, 48, 59-60). Algunos autores encuentran que 
ORIGINALES Y REVISIONES

la memoria prospectiva basada en indicios temporales está también afectada en los niños con TEA (61-62).

"Deseo obsesivo de invariabilidad": las funciones ejecutivas en los Trastornos del Espectro Autista:

M. Lezak define las funciones ejecutivas como las capacidades mentales esenciales para llevar a cabo una conducta eficaz, creativa y socialmente aceptada. Otras definiciones más recientes de este constructo incluyen funciones como planificación, memoria de trabajo, inhibición, flexibilidad cognitiva, monitorización, branching, toma de decisiones etc (63). Las funciones ejecutivas están implicadas en tareas cotidianas y son fundamentales para una adaptación exitosa, motivo por cual han sido objeto de un considerable número de publicaciones.

En relación a éstas, Leo Kanner describe en su artículo de 1943 un "deseo obsesivo de invariabilidad" y "limitaciones en la variedad de actividades espontáneas", además de "dificultades para iniciar interacciones sociales". Podemos considerar que estos enunciados aluden a dificultades en algunas funciones ejecutivas; y que los resultados en algunas pruebas de medida de estas funciones serán también inferiores a los esperados. Una vez más, los resultados de los trabajos revisados son contradictorios.

El Wisconsin Card Sorting Test- WCST (64) es una de las pruebas más empleadas para medir flexibilidad cognitiva, función relacionada con el córtex prefrontal dorsolateral. Cabría esperar que, en base a la rigidez característica observada en los casos de TEA, estas manifestaciones se trasladasen al contexto experimental y los resultados en esta prueba fuesen significativamente inferiores a los del grupo control; pero mientras que algunos trabajos encuentran un rendimiento normalizado empleando el Wisconsin Card Sorting Test o pruebas de formato similar (65-66), otros autores sí lo describen como deficitario (67). Russel Smith et al (2014) emplean una tarea similar al WCST y encuentran un rendimiento similar en los TEA y pares sin este diagnóstico, pero destacan que, frente al empleo de estrategias de monitorización y autoinstrucciones presentes en los controles, los sujetos con TEA no se benefician de éstas (68).

Pero más allá de describir únicamente el rendimiento en algunos test, algunos trabajos intentan potenciar la validez externa de dichas pruebas, y encuentran que los resultados de los test de funciones ejecutivas correlacionan con las medidas de conductas repetitivas y estereotipadas (69-70), con déficit en lenguaje (71), con funcionamiento social y comunicativo (72), o con habilidades de mentalización, obteniéndose resultados opuestos en esta última (73-74). 
ORIGINALES Y REVISIONES

Al igual que en el estudio de la atención y de la memoria, varios autores se han interesado por comparar el rendimiento de niños y adolescentes con TEA en pruebas de medida de funciones ejecutivas con el que se demuestran menores con otros diagnósticos. Mientras que los sujetos con Trastorno por Déficit de Atención e Hiperactividad tienen rendimiento superior al observado en los sujetos con TEA $(51,75)$; aquellos diagnosticados con Trastorno Obsesivo Compulsivo tienen un peor control inhibitorio (76), y Verté et al (2005) encuentran que la flexibilidad cognitiva en TEA es inferior a la que demuestran los menores con Trastorno de Tourette (77). Por otra parte demuestran un mejor rendimiento en pruebas de habilidades visoespaciales en comparación con aquellos con diagnóstico del conocido como Trastorno del Aprendizaje Procedimental (78); mientras que el rendimiento en pruebas de función ejecutiva es similar entre menores diagnosticados de autismo y aquellos con otro diagnóstico de Trastorno Generalizado del Desarrollo en terminología del anterior DSM-IV-TR (79), resultado que podría categorizarse como otra evidencia más a favor de la actual integración de todos ellos bajo la denominación TEA.

\section{Consideraciones finales.}

El análisis de trabajos centrados en la atención, memoria y funciones ejecutivas en los TEA refleja resultados opuestos, incluso en trabajos en los que se emplean las mismas pruebas de evaluación. Habiendo controlado las variables nivel cognitivo y nivel de desarrollo del lenguaje en todos los estudios analizados, la disparidad de resultados puede explicare en base al diferente rango de edad en las muestras de los estudios o al empleo de diferentes pruebas de evaluación. Queda patente sin embargo, que independientemente de estas posibles fuentes de sesgo, el rendimiento de los menores en TEA en las pruebas pone de manifiesto dificultades para cambiar el foco atencional entre estímulos o tareas, preferencia atencional por objetos frente a rostros y menor desarrollo de la atención conjunta. En cuanto a la memoria, si bien no se puede concluir que exista un déficit mnésico global, parecen tener dificultades en el empleo de estrategias de codificación del material, con una repercusión negativa tanto en la fase de almacenamiento como en la de recuperación de la información, además de pobres habilidades de memoria ejecutiva y peor rendimiento en las tareas de memoria episódica (recuerdo de eventos autobiográficos). Los trabajos que analizan flexibilidad cognitiva, monitorización y memoria prospectiva son también negativos en cuanto al resultado de los menores con TEA.

Por tanto, el perfil patológico que Leo Kanner describió a partir de la observación conductual de sus pacientes se confirma con los resultados encontrados en trabajos actuales, y empleando diseños experimentales y medidas mucho más sofisticadas. 
Se incluyen aquí los déficit relativos a atención y funciones ejecutivas (que Kanner relacionó con inflexibilidad y dificultades manifiestas en contextos de interacción social), así como en los componentes más ejecutivos de la memoria (aspecto que Kanner no había precisado en la descripción inicial). Frente al supuesto fundamental de que únicamente los resultados de una evaluación no permiten concluir una afectación cognitiva determinada (ya sea atención, memoria o funciones ejecutivas como la planificación), es imprescindible tener en cuenta varias fuentes de información y contextualizar toda esa información en el marco individual y característico del paciente; característica que sí ha sido obviada en varios trabajos al carecer de otras medidas funcionales. Leo Kanner sentó las bases de lo que en la actualidad se conocen como TEA, y si bien se ha avanzado mucho y ampliado el conocimiento desde sus primeras ideas, es imprescindible que todos los futuros avances en este campo estén guiados por criterios de funcionalidad y utilidad para el paciente.

\section{BIBLIOGRAFÍA:}

(1) Kanner, L Autistic disturbances of affective contact. Nervous Child.1943; 2: 217-250.

(2) Kanner L.The conception of wholes and parts in early infantile autism. Am J Psychiatry 1951; 108: $23-6$

(3) Bettelheim B. The Empty Fortress: Infantile Autism and the Birth of the Self. New York: The Free Press, 1967.

(4) Artigas Pallarés J y Paula I. El autismo 70 años después de Leo Kanner y Hans Asperger. Revista de la Asociación Española de Neuropsiquiatría.2012; 32 (115): 567-587.

(5) Wing L. The definition and prevalence of autism: Areview. Eur ChildAdolesc Psychiatry.1993; 2:61-74.

(6) American Psychiatric Association. Manual diagnóstico y estadístico de los trastornos mentales (DMS-V). Quinta edición. Barcelona: Masson, 2013.

(7) Damasio AR, Maurer RG. A neurological model for childhood autism. Arch Neurol. 1978; 35(12):777-786.

(8) Pina-Camacho L, Villero S, Fraguas D, Boada L, Janssen J, Navas-Sánchez FJ et al. Autism spectrum disorder: Does neuroimaging support the DSM-5 proposal for a symptom dyad? A systematic review of functional magnetic resonance imaging and diffusion tensor imaging studies. J Autism Dev Disord. 2012; 42(7): 1326-41.

(9) O’Hearn K, Asato M, Ordaz S, Luna B. Neurodevelopment and executive function in autism. Dev Psychopathol. 2008; 20(4):1103-1132.

(10) Happé F, Frith U. The Neuropsychology of Autism. Brain.1996; 119: 1377-1400.

(11) Solbergh MM, MateerCA. Management of Attention Disorders. En: Cognitive Rehabilitation. An Integrative Neuropsychological Approach. Londres: Guilford Press; 2001.p. 125-161.

(12) May T, Cornish K, Rinehart N. Does gender matter? A one year follow-up of autistic, attention and anxiety symptoms in high-functioning children with autism spectrum disorder. J Autism Dev Disord. 2014; 44(5):1077-1086.

(13) Landry R, Bryson S E. Impaired disengagement of attention in young children with autism. J Child Psychol Psychiatry. 2004; 45(6): 1115-1122. 
(14) Sasson N J, Elison J T, Turner-Brown LM, Dichter G S, Bodfish J W. Brief report: Circumscribed attention in young children with autism. J Autism Dev Disord. 2011; 41(2): 242-247.

(15) Kikuchi Y, Senju A, Tojo Y, Osanai H, Hasegawa T. Faces do not capture special attention in children with autism spectrum disorder: A change blindness study. Child Dev. 2009; 80(5):1421-1433.

(16) Sasson N J, Touchstone E W. Visual attention to competing social and object images by preschool children with autism spectrum disorder. J Autism Dev Disord. 2014; 44(3): 584-592

(17) Amso D, Haas S, Tenenbaum E, Markant J, Sheinkopf SJ. Bottom-up attention orienting in young children with autism. J Autism Dev Disord. 2014; 44(3):664-673

(18) Jiang YV, Capistrano CG, Esler AN, Swallow KM. Directing attention based on incidental learning in children with autism spectrum disorder. Neuropsychology.2013; 27(2):161-169.

(19) Johnson KA, Robertson I H., Kelly SP, Silk TJ, Barry E, Dáibhis A et al. Dissociation in performance of children with ADHD and high-functioning autism on a task of sustained attention. Neuropsychologia. 2007; 45(10): 223-2245.

(20) Rao PA.; Landa R J. Association between severity of behavioral phenotype and comorbid attention deficit hyperactivity disorder symptoms in children with autism spectrum disorders. Autism. 2014; 18(3): 272-280.

(21) Yerys B E, Wallace G L, Sokoloff JL, Shook DA, James J D, Kenworthy L. Attention deficit/ hyperactivity disorder symptoms moderate cognition and behavior in children with autism spectrum disorders. Autism Res. 2009; 2(6): 322-333

(22) Mattard-Labrecque C, Amor LB, Couture M M. Children with autism and attention difficulties: A pilot study of the association between sensory, motor, and adaptive behaviors. J Can Acad Child Adolesc Psychiatry. 2013; 22(2):139-146.

(23) Hanley M, Riby DM, McCormack T, Carty C, Coyle L, Crozier N et al. Attention during social interaction in children with autism: Comparison to specific language impairment, typical development, and links to social cognition. Res Autism Spectr Disord. 2014; 8(7): 908-924.

(24) Parish-Morris J, Chevallier C, Tonge N, Letzen J, Pandey J; Schultz, RT. Visual attention to dynamic faces and objects is linked to face processing skills: A combined study of children with autism and controls. Front Psychol. 2013; 4.

(25) Jarrold W, Mundy P, Gwaltney M, Bailenson J, Hatt N, McIntyre N et al. Social attention in a virtual public speaking task in higher functioning children with autism. Autism Res. 2013; 6(5): 393-410

(26) Bar-Haim Y, Shulman C, Lamy D, Reuveni A. Attention to Eyes and Mouth in HighFunctioning Children with Autism. J Autism Dev Disord. 2006; 36(1):131-137.

(27) Bayram S, Esgin E. Patterns of visual attention and gaze to human and animal faces in children with Autism Spectrum Disorders. World Journal on Educational Technology. 2012; 4(3):165-179.

(28) Deruelle C, Rondan C, Salle-Collemiche X, Bastard-Rosset D, Da Fonséca D. Attention to low- and high-spatial frequencies in categorizing facial identities, emotions and gender in children with autism. Brain Cogn. 2008; 66(2):115-123.

(29) Begeer S, Rieffe C, Terwogt MM, Stockmann L. Attention to facial emotion expressions in children with autism. Autism. 2006; 10(1): 37-51.

(30) Bruinsma Y, Koegel RL, Koelen LK. Joint Attention and Children with Autism: A Review of the Literature. Ment Retard Dev Disabil Res Rev. 2004; 10(3): 169-175.

(31) Goldberg MC, Moston AJ, Vecera S P, Larson J C, Mostofsky SH.; Mahone EM et al. Evidence for impairments in using static line drawings of eye gaze cues to orient visual-spatial attention in children with high functioning autism. J Autism Dev Disord. 2008; 38(8): 1405-1413.

(32) Kylliäinen A, Hietanen JK. Attention orienting by another's gaze direction in children with autism. Journal of Child Psychology and Psychiatry.2004; 45(3):435-444. 
(33) Murray D S, Creaghead NA,Manning-Courtney P, Shear PK, Bean J,Prendeville JA. The relationship between joint attention and language in children with autism spectrum disorders. Focus on Autism and Other Developmental Disabilities.2008; 23(1):5-14.

(34) Wong C, Kasari C. Play and joint attention of children with autism in the preschool special education classroom. J Autism Dev Disord. 2012; 42(10): 2152-2161.

(35) Swanson M R, Siller M. Patterns of gaze behavior during an eye-tracking measure of joint attention in typically developing children and children with autism spectrum disorder. Research in Autism Spectrum Disorders.2013; 7(9):1087-1096.

(36) Swanson MR, Serlin GC, Siller M. Broad autism phenotype in typically developing children predicts performance on an eye-tracking measure of joint attention. J Autism Dev Disord. 2013; 43(3):707718.

(37) Chawarska K, Klin A, Volkmar F. Automatic Attention Cueing Through Eye Movement in 2-Year-Old Children With Autism. Child Dev. 2003; 74(4):1108-1122.

(38) Warreyn P, Roeyers H, Oelbrandt T, De Groote I. What Are You Looking at?: Joint Attention and Visual Perspective Taking in Young Children With Autism Spectrum Disorder. Journal of Developmental and Physical Disabilities.2005; 17(1): 55-73.

(39) Sanna Kuusikko-Gauffin E, Jansson-Verkasalo A, Carter R, Pollock-Wurman K, Jussila M $\mathrm{L}$, Mattila J et al. Face memory and object recognition in children with high-functioning autism or Asperger syndrome and in their parents. Research in Autism Spectrum Disorders.2011; 5(1): 622-628

(40) Phelan H L, Filliter JH, Johnson SA. Brief report: Memory performance on the California Verbal Learning Test - Children's Version in autism spectrum disorder. J Autism Dev Disord. 2011; 41(4):518-523

(41) Renner PK, Grofer Klinger L, Mark R. Implicit and explicit memory in autism: Is autism an amnesic disorder?. J Autism Dev Disord. 2000; 30(1): 3-14

(42) Southwick JS, Bigler ED, Froehlich A, DuBray M B, Alexander AL, Lange N, Lainhart JE. Memory functioning in children and adolescents with autism. Neuropsychology.2011; 25(6):702-710.

(43) Lind S E, Bowler D M. Recognition memory, self-other source memory, and theory-of-mind in children with autism spectrum disorder. J Autism Dev Disord. 2009; 39(9): 1231-1239.

(44) Goddard L, Dritschel B, Robinson S, Howlin P. Development of autobiographical memory in children with autism spectrum disorders: Deficits, gains, and predictors of performance. Dev Psychopathol. 2014; 26(1): 215-228.

(45) Andersen PN, Hovik KT, Skogli EW, Egeland J, Oie M. Symptoms of ADHD in children with high-functioning autism are related to impaired verbal working memory and verbal delayed recall. PLoS ONE.2013; 8(5): e64842

(46) Bruck M, London K, Landa R, Goodman J. Autobiographical memory and suggestibility in children with autism spectrum disorder. Dev Psychopathol. 2007; 19(1): 73-95.

(47) Lind S E. Memory and the self in autism. A review and theoretical framework. Autism, 2010. 14 (5): 430-456.

(48) Millward C, Powell S, Messer D, Jordan R. Recall for self and other in autism: Children's memory for events experienced by themselves and their peers. J Autism Dev Disord. 2000; 30(1): 15-28.

(49) Hill E L, Russell J. Action memory and self-monitoring in children with autism: Self versus other. Infant and Child Development. Special Issue: Executive functions and development: Studies of typical and atypical children. 2002; 11(2):159-170.

(50) Jiang Y V, Capistrano CG, Palm B E. Spatial working memory in children with highfunctioning autism: Intact configural processing but impaired capacity. J Abnorm Psychol. 2014;123(1):248257. 
(51) Zinke K, Fries E, Altgassen M, Kirschbaum C, Dettenborn L, Kliegel M. Visuospatial shortterm memory explains deficits in tower task planning in high-functioning children with autism spectrum disorder. Child Neuropsychol.2010; 16(3): 229-241.

(52) Geurts H M, Verté S, Oosterlaan J, Roeyers H, Sergeant JA. How specific are executive functioning deficits in attention deficit hyperactivity disorder and autism?.J Child Psychol Psychiatry.2004; 45(4): 836-854.

(53) Landa RJ, Goldberg M C. Language, Social, and Executive Functions in High Functioning Autism: A Continuum of Performance. J Autism Dev Disord. 2005 35(5): 557-573

(54) Mammarella I C, Giofrè D, Caviola S, Cornoldi C, Hamilton C. Visuospatial working memory in children with autism: The effect of a semantic global organization. Res Dev Disabil. 2014; 35(6): 1349-1356.

(55) Gabig C S. Verbal working memory and story retelling in school-age children with autism. Lang Speech Hear Serv Sch. 2008; 39(4): 498-511.

(56) Englund J A, Decker S L, Allen R A, Roberts A M. Common cognitive deficits in children with attention-deficit/hyperactivity disorder and autism: Working memory and visual-motor integration. Journal of Psychoeducational Assessment. 2014; 32(2): 95-106.

(57) De Noreña D, Maestú F. Neuropsicología de la memoria. En: Tirapu Ustárroz J, Ríos Lago M, Maestú Unturbe F (Eds). Manual de Neuropsicología. 2a Ed. Barcelona: Viguera, 2011; p. 191-217.

(58) Wojcik D Z, Moulin C J A, Souchay C. Metamemory in children with autism: Exploring "feeling-of-knowing" in episodic and semantic memory. Neuropsychology.2013; 27(1): 19-27.

(59) Joseph RM, Steele S D, Meyer E, Tager-Flusberg H. Self-ordered pointing in children with autism: Failure to use verbal mediation in the service of working memory? Neuropsychologia. 2005; 43(10): $1400-1411$.

(60) Goldman S. Brief report: Narratives of personal events in children with autism and developmental language disorders: Unshares memories. J Autism Dev Disord. 2008; 38 (10): 1982-1988.

(61) Altgassen M, Williams T I, Bölte S, Kliegel M. Time-based prospective memory in children with autism spectrum disorder. Brain Impairment. Special Issue: Prospective memory 2009; 10(1): 52-58.

(62) Yi L, Fan Y, Joseph L, Huang D, Wang X, Li J, Zou X. Event-based prospective memory in children with autism spectrum disorder: The role of executive function. Res Autism Spectr Disord.2014; 8(6): 654-660.

(63) Gómez Beldarrain M, Tirapu Ustárroz J. Neuropsicologia de la corteza prefrontal y las funciones ejecutivas: una visión panorámica. En: Tirapu Ustárroz J, García Molina A, Ríos Lago My Ardila Ardila A (Eds). Neuropsicología de la corteza prefrontal y las funciones ejecutivas. Barcelona: Viguera, 2012; p. 1-14.

(64) Heaton R K, Chelune G J, Talley J L, Kay G G y Curtiss G. WCST. Test de Clasificación de Tarjetas de Wisconsin (adaptación española de M. ${ }^{a}$ Victoria de la Cruz (Dpto. de I+D+i de TEA Ediciones).1997. Madrid: TEA Ediciones.

(65) Maister L, Simons J S, Plaisted-Grant K. Executive functions are employed to process episodic and relational memories in children with autism spectrum disorders. Neuropsychology.2013; 27(6): 615-627

(66) Robinson S, Goddard L, Dritschel B, Wisley M, Howlin P. Executive functions in children with autism spectrum disorders. Brain Cogn. 2009; 71(3): 362-368.

(67) Semrud-Clikeman M, Goldenring Fine J, Bledsoe J. Comparison Among Children with Children with Autism Spectrum Disorder, Nonverbal Learning Disorder and Typically Developing Children on Measures of Executive Functioning High Functioning Autism: A Continuum of Performance. J Autism Dev Disord. 2014; 44: 331-342. 
(68) Russell-Smith S N, Comerford B J. E, Maybery M T, Whitehouse A J. O. Brief report: Further evidence for a link between inner speech limitations and executive function in high-functioning children with autism spectrum disorders. J Autism Dev Disord. 2014; 44(5): 1236-1243.

(69) Boyd BA, McBee M, Holtzclaw T, Baranek G T, Bodfish J W. Relationships among repetitive behaviors, sensory features, and executive functions in high functioning Autism. Res Autism Spectr Disord. 2009; 3(4): 959-966.

(70) South M, Ozonoff S, McMahon WM. The relationship between executive functioning, central coherence, and repetitive behaviors in the high-functioning autism spectrum. Autism. 2007; 11(5): 437-451.

(71) Kenworthy L, Black D O, Harrison B, Della Rosa A, Wallace G L. Are executive control functions related to autism symptoms in high-functioning children?. Child Neuropsychol. 2009; 15(5): 425440 .

(72) Gilotty L, Kenworthy L, Sirian L, Black DO, Wagner AE Adaptive skills and executive function in autism spectrum disorders. Child Neuropsychol. 2002; 8(4): 241-248.

(73) Joseph R M, Tager-Flusberg H. The relationship of theory of mind and executive functions to symptom type and severity in children with autism. Dev Psychopathol. 2004; 16(1): 137-155.

(74) Pellicano E. Links between theory of mind and executive function in young children with autism: Clues to developmental primacy. Dev Psychol. 2007; 43(4): 974-990.

(75) Happe' F, Booth R, Charlton R, Hughes C. Executive function deficits in autism spectrum disorders and attention-deficit/hyperactivity disorder: Examining profiles across domains and ages. Brain Cogn. 2006; 61: 25-39.

(76) Zandt F, Prior M, Kyrios M. Similarities and differences between children and adolescents with autism spectrum disorder and those with obsessive compulsive disorder: Executive functioning and repetitive behaviour. Autism. 2009; 13(1): 43-57.

(77) Verté S, Geurts H M, Roeyers H, Oosterlaan J, Sergeant J A. Executive functioning in children with autism and Tourette syndrome. Dev Psychopathol. 2005; 17(2): 415-445.

(78) Semrud-Clikeman M, Fine J G, Bledsoe J. Comparison among children with children with autism spectrum disorder, nonverbal learning disorder and typically developing children on measures of executive functioning. J Autism Dev Disord. 2014; 44(2): 331-342.

(79) Rosenthal M, Wallace G L, Lawson R, Wills M C, Dixon E, Yerys B E et al. Impairments in real-world executive function increase from childhood to adolescence in autism spectrum disorders. Neuropsychology. 2013; 27(1): 13-18. 